\title{
The effective use of a decision support tool in the area of strategic marketing
} management.

\author{
P.R.Burrell
}

Knowledge Base Systems Centre, South Bank University, Borough Road, London SE1 0AA, UK, email: phillb@vax.sbu.ac.uk.

\section{Y. Duan}

Faculty of Business, University of Luton, Luton LU1 3JU, UK, email: duany@vax2.luton.ac.uk.

\section{A. Boskovic}

LAFORIA-IBP, University Paris VI,UPMC, BP 169, 75252 Paris, cedex 5, France.

\begin{abstract}
Management at a strategic level involves a high level of risk and uncertainty. These problems are compounded further in the area of marketing due to the fact that firms often face turbulent and unpredictable markets. Expert systems and decision support systems might have a large impact in this area but the development of such systems has proven difficult with less than expected results. This paper provides a description of an effective decision support tool embedded within a hybrid expert/decision support system. This tool is derived from a modified version of an existing operational research technique and is used to introduce structure and objectivity into a largely subjective process of attaching values to a set of decision criteria.
\end{abstract}

Keywords

Analytic hierarchy process, decision support, expert system, strategic management, marketing

\section{INTRODUCTION}

Managers at the strategic level are responsible for the "external and future" and long term plans. Such decisions are frequently high risk with a considerable degree of uncertainty attached to the outcomes. The knowledge needed at this level is not as specific as that in the lower level of a company and, by nature, is more unstructured. Beerel (1987) points out that strategic managers are continuously faced with complex and unstructured decisions at a high level of uncertainty and have a lot at stake. The strategic manager mediates the relations between the company and the outside world and they are responsible for the general direction of the company. The relationship of the company to its 
environment is a central matter of concern and predictions about the future are particularly important. The information required is aggregate information. Both the scope and variety of the information are quite large, but the requirements for accuracy are not particularly stringent.

This level of uncertainty applies, probably more than most, to areas of marketing and as most firms have to face turbulent and sometimes unpredictable markets, their managers have to watch and decide about their product-market strategy. Nobody should doubt the importance of marketing planning in the modern business. However, Kotler (1991) finds that many companies, for various reasons, operate without plans. He stresses that formal planning can yield many benefits for all types of companies. Sound planning helps the company to anticipate and respond quickly to environmental changes, and to prepare for sudden developments.

Although a huge amount of information is available on marketing planning, it is still an area which is less well understood and where there is a lack of a framework which can be easily transferred into a computer system, or directly applicable to build an expert system (ES). As Mitchell et al (1991) have found, our understanding of causal structure that relates market factors is relatively loose, at least compared to the engineering domain in which many expert systems have been developed. The development of ESs has revealed the incompetence of our own knowledge of how to analyse marketing problems. Because of these reasons the construction of ESs in marketing is more difficult than in other domains.

The authors (Duan and Burrell, 1995a) believe that these difficulties arise because traditional ES technology is too inflexible to cope with the loose causal structure of information associated with the marketing domain. For this reason, a more flexible approach in the form of a hybrid system combining both expert system and decision support technology was required. Not only was there a need for a hybrid technology but also the need for appropriate decision support tools capable of working within the framework of the marketing domain. This paper presents a brief introduction to HYMS [HYbrid Marketing System] in order to provide an understanding of the core model used and the issues related to its use. It then discusses, in more detail, the problems associated with structuring the domain like marketing and how an operation research technique, the Analytic Hierarchy Process was modified, in order to overcome its deficiencies, and used to establish objectivity and structure in the decision hierarchy.

\section{INTRODUCTION TO HYMS}

The system architecture of the initial prototype is shown in Figure 1. The system consists of several modules, some of which contain knowledge bases, and others, decision support. The system guides the user through the marketing planning process form the pre-diagnostic stage, by establishing goals and supplying information where necessary, to providing advice on the best strategy to adopt. A fuller description of HYMS has been presented by the authors (Duan and Burrell, 1995b, 1996) in other publications. 


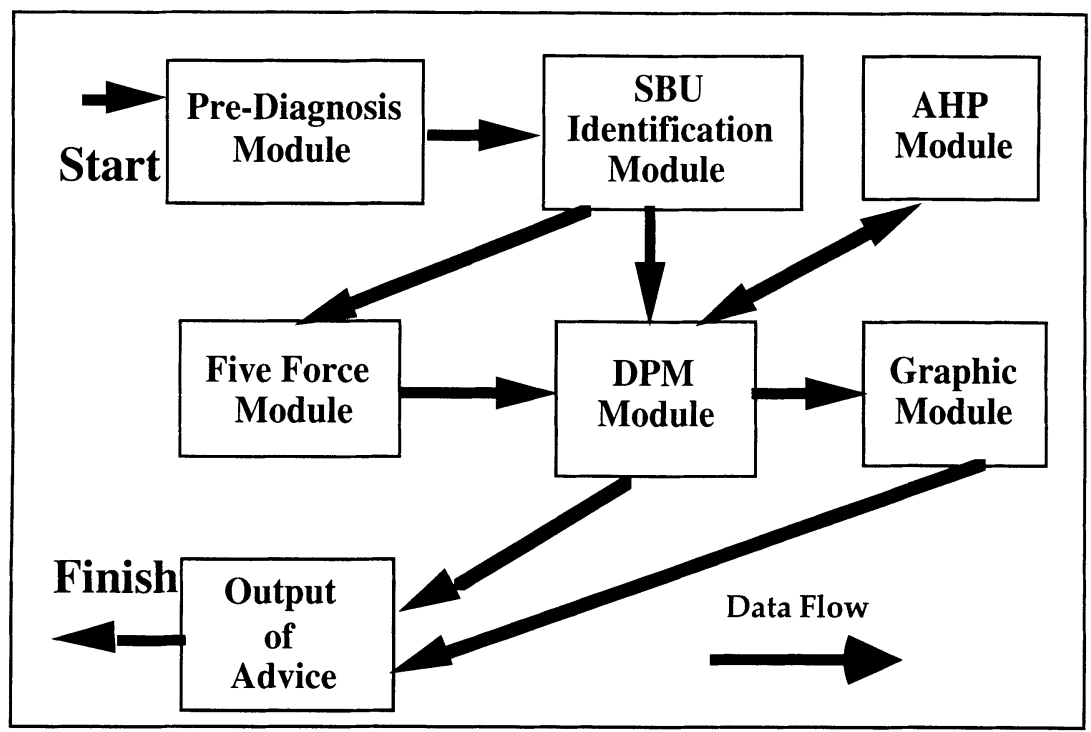

Figure 1 System Architecture of HYMS

The core module of HYMS is the Directional Policy Matrix (DPM) which consists of three components; the Market Strength Evaluator, the Business Strength Evaluator and the Strategy Generator. The DPM is based on McDonald's (1989) and McDonald and Wilson's (1990) nine step marketing planning process model and is implemented as a $3 * 3$ matrix where a company's strategic business unit is assessed, based on factors related to the business strength and market attractiveness. The dimensions used for assessing the position on the matrix represent the significant factors of the internal and external environment from which strengths, weaknesses, opportunities and threats arise. Nine boxes are formulated and each of these positions calls for a different marketing strategy. Qualitative analysis is used to assess the business strength as strong, medium or weak and the market attractiveness as high, medium or low.

The qualitative analysis for the DPM is implemented through the Analytic Hierarchy Process (AHP) module by applying a method of pair-wise comparison to the factors related to business strength and market attractiveness. This process results in a set of weights being attached to these factors which is then used by the strategy generator to plot a position on the policy matrix.

\section{THE DECISION HIERARCHY}

One of the most important aspects governing any decision support is choosing the factors related to the decision process and then arranging them in a hierarchical structure. Once selected, these are arranged in order descending from an overall goal, to criteria, to sub-criteria and to any alternatives at successive levels. Arranging the goals in a hierarchy serves the purposes of providing an overall view of the complex relationships inherent in a situation 
and helps the decision maker assess whether the issues in each level are of the same order of magnitude.

Implementing this decision hierarchy for decision support in the area of diagnostics is reasonably straight forward, in the way that top down goal decomposition can be applied to a majority of problems. In the area of strategic marketing planning this is not the case, as the task is compounded by the fact that this hierarchy is not a traditional decision tree. Each of the factors may represent a different cut of the problem. Some may represent social factors while another, economic factors. Also, both of these may need to be evaluated in terms of the other. It may also be necessary to insert or eliminate factors as necessary to clarify the task of setting priorities or to sharpen the focus on one or more parts of the hierarchy. Thus a hierarchy representing a general problem can be easily adapted to represent one specific case.

Although the problem of structuring the decision hierarchy for the given application is subject to the constraints mentioned, it transpires that the decision process relies more on the relative importance assigned to each of the factors of the decision hierarchy. This allows a certain amount of flexibility in the design of the hierarchy in that it was not necessary to establish a causal relationship between the factors in the hierarchy, but more to group related factors and sub factors together. The top level factors were dictated by the use of the chosen DPM model and the sub factors relating to these were positioned accordingly. These are shown in Figure $2 \mathrm{a}, 2 \mathrm{~b}$.

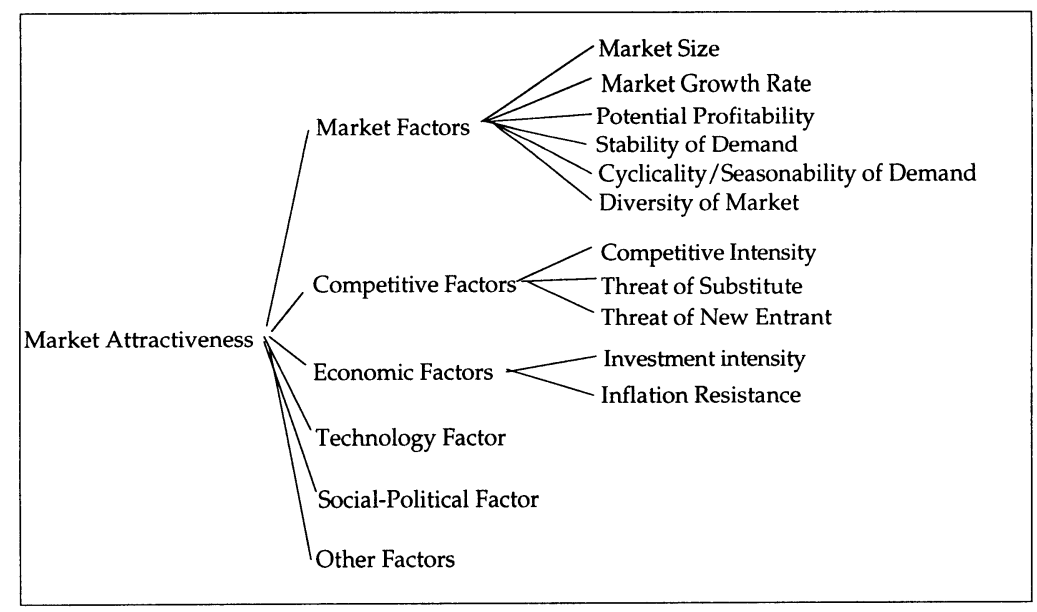

Figure 2a The hierarchies of decision factors for market attractiveness

The decision hierarchy was established from knowledge collected from experts and relevant documents. Using published documents, as a major source of knowledge, is quite common in the marketing area. COMSTRAT (Curry et al, 1992) (Mentzer and Gandhi, 1992) and NEGOTEX (Rangaswamy et al, 1989) used the relevant academic literature to generate their decision hierarchies and McDonald (1989), and McDonald and Wilson (1990) have published papers and 
reports about his marketing planning model. Although the knowledge from published documentation is easy to access, it is limited to generalities and is not sufficient to build a specific hierarchy. It was therefore deemed necessary to elicit the help of experts as another major source for knowledge.

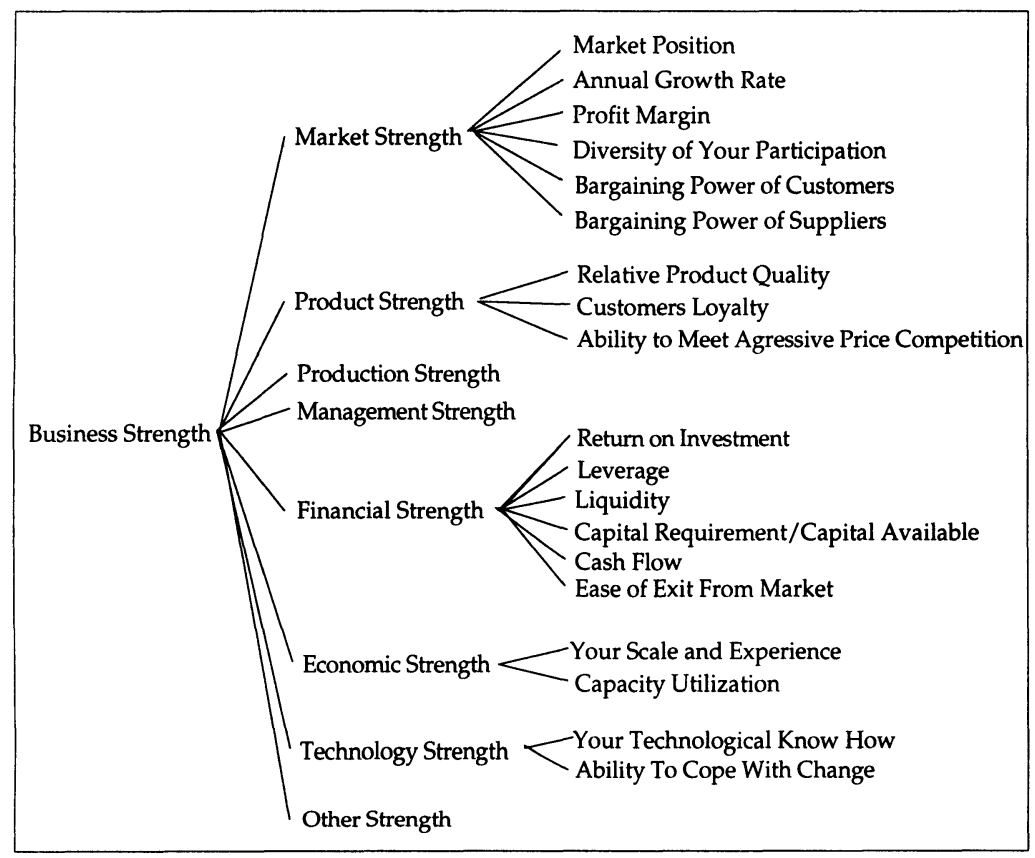

Figure 2b The hierarchies of decision factors for business strength

\section{THE AHP PROCESS}

Having established a structure for the decision hierarchy it becomes necessary to assign some value of importance to the factors composing the hierarchy. This raises the question as to whether each of the factors/sub-factors should be of equal importance. As Day (1986) indicated, in relation to the marketing domain, there are several problems: first, many of the factors are interrelated; second, a factor such as technological position may be of minor significance in one market while being the dominant determinant of survival in another market swept by a new generation of technology. Assigning values to various factors entirely depends on different circumstance and users' personal judgement. Any decision support capable of satisfying these requirements must be able to introduce structure and objectivity into the largely subjective process of attaching values to a set of decision criteria in a multi-criteria decision making situation.

The chosen model to implement this subjective process was the AHP. This is an Operational Research (OR) technique for applications in diverse decision problem areas (Saaty, 1977, 1978, 1980, 1990) and is concerned with how to derive 
relative scales using judgements given in the form of pair-wise comparisons. In this instance, the pair-wise comparisons are carried out between the factors and sub-factors related to the decision hierarchies of both the market attractiveness and business strength.

Solving a decision problem, using the AHP, involves four steps (Johnson 1980):

- Establishing a decision hierarchy.

- The pair-wise comparison of the decision elements.

- Estimating the relative weights of the deciding elements using the "eigenvalue method".

- Aggregating the relative weights of decision elements to be applied at different levels of the decision hierarchy.

It was only necessary to implement the first three of these steps as the complexity of the full AHP was not required. The result of the eignvalue method gives a matrix with the following form:

$A^{*} \mathrm{~W}=\mathrm{n}^{*} \mathrm{~W}$

where $A$ is the is the matrix of pair-wise comparisons, $W=\left(w_{1}, w_{2}, \ldots \ldots . ., w_{n}\right)^{T}$ being the vector of relative weights; the eigenvector, and $n$ the number of elements; the eigenvalue. Because the pair-wise values of matrix A rely upon human judgement, matrix $\mathrm{A}$ contains inconsistencies and therefore $\mathrm{n}$ may only be considered as an approximation. This now becomes:

$\underline{\mathrm{A}}^{*} \underline{\mathrm{W}}=\operatorname{lmax}^{*} \underline{\mathrm{W}}$

where $\underline{A}$ is the observed matrix of pair-wise comparisons, $l_{\max }$ is the principle eigenvalue and $\underline{W}$ is an estimation of $W$. The closer $l_{\max }$ is to $n$, the more consistent are the pair-wise values of $\underline{\mathrm{A}}$. This has led to the need of a consistency index (CI) Saty (1980) where:

$\mathrm{CI}=\left(\mathrm{l}_{\max }-\mathrm{n}\right) /(\mathrm{n}-1)$

and the consistency ratio (CR):

$\mathrm{CR}=(\mathrm{CI} / \mathrm{ACI})^{*} 100$

where the Average Consistency Ratio (ACI) is the average index of randomly generated weights. Saaty (1980) proposes the rule of thumb, where a CR of $10 \%$ or less is considered acceptable. If this is not the case, then the pair-wise comparison of $\underline{\mathrm{A}}$ needs to be recalculated. The value of $\mathrm{ACI}$ depends upon the size of the matrix and is shown in Figure 3.

\begin{tabular}{lllllllll} 
& $\mathbf{n}$ & 2 & 3 & 4 & 5 & 6 & 7 & 8 \\
\hline ACI & 0.00 & 0.58 & 0.90 & 1.12 & 1.24 & 1.32 & 1.41 &
\end{tabular}

Figure 3 The ACI for a linear 1-9 scale (Saaty's result) 


\section{DEFICIENCIES WITH THE AHP}

Criticisms regarding the use of the AHP seemed to be levelled at the use of the linear scale of comparisons. This is a standard scale used for establishing the difference of importance between elements in the decision hierarchy. Saaty (1980) describes the scale as linguistic and supports its use through a series of experiments. This scale is shown in Figure 4.

Intensity of importance

Definition

Explanation

1

3

5

7

9

$2,4,6,8$

Reciprocals
Equal importance

Weak importance of one over another

Essential or strong importance of one over another

Demonstrated importance

Absolute, extreme importance

Intermediate values between two adjacent judgements
Two activities contribute equally to the objective

Experience and judgement slightly favour one activity over another

Experience and judgement strongly favour one activity over another

An activity is strongly favoured and its dominance is demonstrated in practice

The evidence favouring one activity over another is of the highest possible order of affirmation

When compromise is needed

If activity $i$ has one of the non zero numbers assigned to it when compared with activity $j$, then $j$ has the reciprocal value when compared with $i$

Figure 4 Saaty's linear scale of comparisons

A review of the major criticisms (Salo and Hamalainen 1994) cited problems in four areas:

- The meaning of the pair wise comparisons.

- The relationship between scores and criteria weights.

- The properties of the 1-9 ratio scale.

- The prohibited complexity of the super-matrix approach.

Davis (1993) also criticised the method as being difficult to use, perhaps because of an unfamiliarity of the scale used. The major concern seems to be the difference between the use of a linguistic scale and the algebraic ratio interpretation of responses implied by its use. A second is the effect of the scale on the inconsistency of the matrix of judgements. In the original version of the AHP the decision maker is presented only with the English language descriptions of the scale. This conceals what is being asked, to estimate ratios of weights for pairs of 
criteria. A number of experiments (Holder, 1990) (Lund and Palmer, 1986), using the nine point linguistic scale, suggested that, dependent upon the users background, the use of a pair-wise ratio presented a clearer interpretation of comparison process and that the use of the linguistic scale often led to an inconsistency of judgements. It is suggested therefore that the use of this scale leads to violations of the normal usage of the English language, for example: If the decision maker evaluates $A$ as weakly more important than B and B as weakly more important than $C$ this implies that $A$ is extremely (absolutely) more important than $\mathrm{C}$.

\section{MODIFICATIONS TO THE AHP}

The way to try to overcome these deficiencies was first, to offer the user a linguistic scale, but not to hide the numerical meaning of the points of the scale. It was considered more "user friendly" to assess intangible, non measurable criteria in English than in pure numbers. However numbers give the linguistic scale a more precise meaning and for that reason, we also presented them to the user. In this way, user evaluation can show if the inconsistency of the judgements is significantly inflated by the use of a linguistic scale. The other deficiency is addressed by using a multiplicative (geometrical) scale. This deficiency was also recognised by Lootsma (1989) who introduced the concept of a symbolic scale based on psychological measurements.

This theory was the bases for the development of a multiplicative scale used in our model. We have adopted a scale with the same number of points as Saaty's, consequently exactly the same semantics have been used. This scale is shown in Figure 5.

\begin{tabular}{|c|c|}
\hline $\begin{array}{l}\text { aij in the matrix of } \\
\text { pair-wise comparisons }\end{array}$ & Semantic interpretation \\
\hline 16 & factor $\mathrm{i}$ is extremely more important than factor $\mathrm{j}$ \\
\hline 8 & factor $i$ is very strongly more important than factor $j$ \\
\hline 4 & factor $i$ is strongly more important than factor $j$ \\
\hline 2 & factor $i$ is moderately more important than factor $j$ \\
\hline 1 & factor $i$ is equally important to factor $j$ \\
\hline $1 / 2$ & factor $\mathrm{i}$ is moderately less important than factor $\mathrm{j}$ \\
\hline $1 / 4$ & factor $\mathrm{i}$ is strongly less important than factor $\mathrm{j}$ \\
\hline $1 / 8$ & factor $i$ is very strongly less important than factor $j$ \\
\hline $1 / 16$ & factor $i$ is extremely less important than factor $j$ \\
\hline
\end{tabular}

Figure 5 The multiplicative scale used in the AHP module

This scale is more satisfactory than the linear 1-9 scale in terms of consistency. Every point of this 1-16 multiplicative scale can be expressed in terms of two other points on the same scale and the "gaps" associated with intermediate values have now been eliminated.

Implementing the 1-16 multiplicative scale as opposed to Saaty's 1-9 linear scale required that the ACI also had to be modified. Saaty's (1980) linear scale integers in the interval 1 to 9 were randomly generated to form a judgement matrix. In 
our model we are using a multiplicative 1 - 16 scale so the corresponding ACI had to be empirically determined. In order to achieve this, and also to test our empirical approach, we first repeated the Saaty's original method, for the 1-9, scale for matrices of size 3 to 8 . For each matrix size the ACI was computed on a sample of 1000 matrices. We found that we had a slight variation in the second decimal digit for some of the points of the scale (Saaty's original scale is shown in Figure 3) but concluded that this was most likely to be due to the distribution of the pseudo-random generator used in the computation. We used the same method to compute the ACI for the multiplicative 1 - 16 scale. The steps of the scale used in the AHP module $(1 / 16,1 / 8,1 / 4, \ldots, 8,16)$ were randomly generated. The sample size of 1000 was not changed. The results were the following, as shown in figure 6.

\begin{tabular}{llllllll} 
& $\mathrm{n}$ & 3 & 4 & 5 & 6 & 7 & 8 \\
\hline $\mathrm{ACI}$ & 0.70 & 1.29 & 1.51 & 1.79 & 1.81 & 2.07
\end{tabular}

Figure 6 The ACI generated for a multiplicative 1-16 scale

These results were used for the consistency check in the AHP module when the comparisons were evaluated on the multiplicative scale.

The original implementation of the AHP requires the decision maker to complete a sequence of positive reciprocal matrices by answering $n(n-1) / 2$ questions for each matrix (where $n$ is the size of the matrix), each entry being an approximation to the ratio of the weights of two items being compared. Harker (1987) developed an extension to the eigenvector approach of the AHP which allows the decision maker to say "I don't know" or "I'm not sure, I prefer not answering" to some of the questions being asked and shows that non negative, quasi-reciprocal matrices can be used in exactly the same manner as positive reciprocal matrices, for which Saaty's AHP theory was developed. Even the relationship between $l_{\max }$ and $n$ remains unchanged. (One still has $l_{\max }>n$ for the general case and $l_{\max }=\mathrm{n}$ iff the matrix $\mathrm{A}$ is consistent). This may be useful when the decision maker does not have a good understanding of their preferences between two particular alternatives or if the alternatives represent a sensitive subject so that the decision maker is unwilling to make a direct comparison. This incomplete pair-wise comparison method was incorporated into our AHP model.

\section{IMPLEMENTATION OF THE MODEL}

The pair-wise comparison of elements is implemented through the AHP module which comprises the AHP Interface Unit and the Eigenvector Computation Unit. The Interface Unit guides the decision maker through the application of the AHP. It presents a graphic display with the hierarchy of factors and sub factors to which the decision maker seeks to assign weights. If necessary, help with the weight assignment can be evoked and is supplied in the form of a sequence of questions to help to evaluate the pair-wise comparisons between all the relevant criteria. The overall view of the evaluations is presented in the form of a triangular matrix. Once the user judges the pair-wise comparisons complete, weights are computed. If all the pair-wise comparisons have not been evaluated, 
then an incomplete judgement matrix is sent to the Eigenvector Computation Unit which treats it as an incomplete pair-wise comparison. A general model of the module shown in Figure 7.

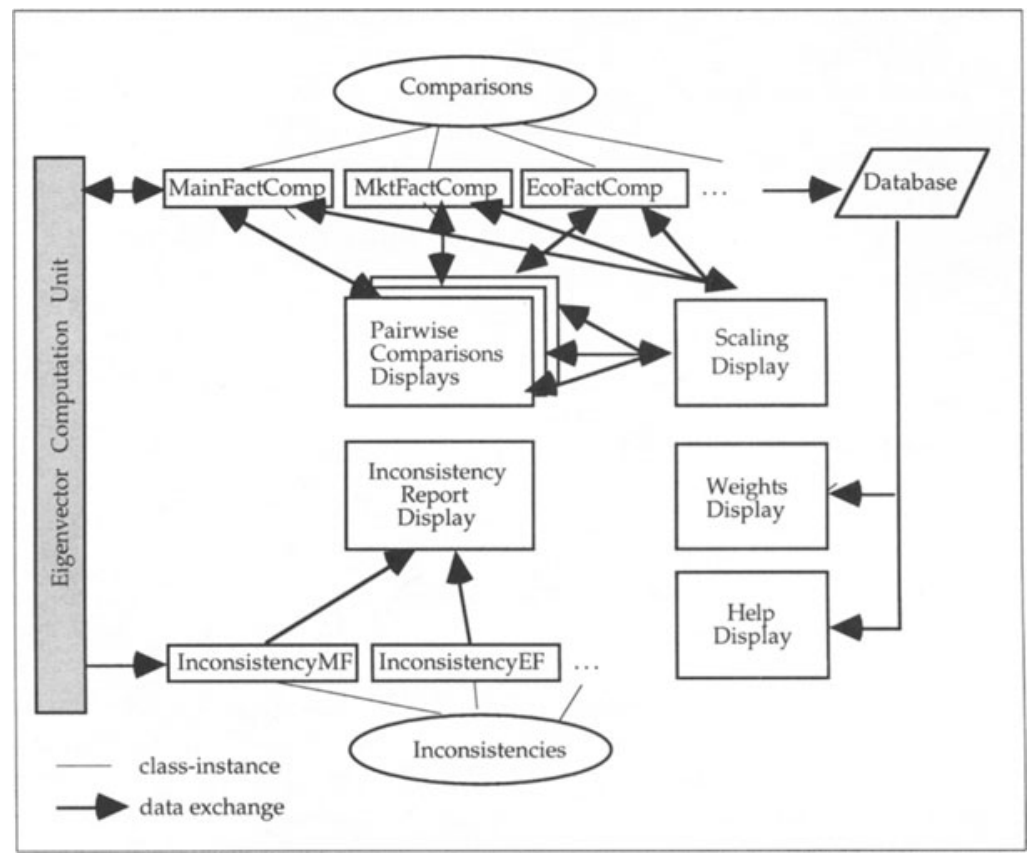

Figure 7 The structure of the AHP Interface Unit

If the user's judgements were consistent, weights are displayed and the user may save them if required. In the case where inconsistencies are detected the AHP Interface Unit generates an Inconsistency Report, from the information provided by the Eigenvector Computation Unit, which gives the details of each triplet of inconsistent comparisons. The inconsistencies are sorted by their magnitudes in a decreasing order and this information can then be used to produce a consistent judgement matrix.

\section{CONCLUSION}

Marketing provides many opportunities for the application of expert systems and decision support systems, but the progress in this area is still relatively slow. This project has shown that HYMS is an effective hybrid decision support tool, and can demonstrate to marketing executives its capability by helping them make more consistent and better decisions. The system developed combines the advantage of ES and DSS. It provides more systematic analysis using selected marketing techniques and appropriate decision support tools.

The use of the AHP has proven an effective method of assigning strengths to various marketing factors in a variety of different marketing situations. 
Although this technique has come under some criticism, these have been largely related to the interpretation of the linguistic used. The modification to this scale have, we believe, have overcome some of these interpretation problems. The use of the 1-16 multiplicative scale now allows the user to express any point on the scale in terms of two other points on the same scale and the qualitative and quantitative values presented to the user provide a method by which more effective judgements can be made for the pair-wise comparisons.

To evaluate the success of such a system as this is a difficult task because there is no objective standard by which any measurements can be made (Wensley 1989). An evaluation of HYMS should cover the system's effectiveness and its usability but the effectiveness and usability of a prototype system, such as this, can really only be evaluated by experts' hands-on experience. The response by a number of experts using the system was generally favourable. The users reported positively on the efficiency and effectiveness of the program. The type, clarity and meaning of conclusions were all deemed acceptable. Furthermore, test conclusions were considered to be accurate "given that this is by nature a fairly inexact science". Most favourable and promising of all was a comment by one user that "the program had proven thought provoking" and had helped him to identify, and challenged him to think about, programmatic areas in his real-life plan.

\section{REFERENCES}

Beerel, A.C. (1987) Expert Systems: Strategic Implications and Applications, Ellis Horwood, Chichester.

Curry, B. Moutinho, L. and Davies, F. (1992) Constructing a knowledge base for a marketing expert system, Marketing Intelligence \& Planning, 10, 12-20.

Day, G.S. (1986) Analysisn for Strategic Market Decisions, West Publising Company, USA.

Davis, A.P. (1993) How an AHP can facilitate Marketing Decision Making, Business School Research Series, Loughborough University, UK.

Duan, Y. and Burrell, P. (1995a) Some Issues in developing Expert Systems for Strategic Marketing Planning, 8th International Symposium on Artificial Intelligence, Monterrey, Mexico, 78-85.

Duan, Y and Burrell, P. (1995b) A hybrid system for strategic market planning, Marketing Intelligence and Planniing, 13, 5-12.

Duan, Y. and Burrell, P. (1996) HYMS: A Hybrid Marketing System,Third World Congress on Expert Systems, Soeul, Korea, Vol. 1, 335-342.

Harker, P.T. (1987) Alternative Modes of Questioning in the AHP, Mathematical Modelling, 9, 353-360.

Holder, R.D. (1990) Some Comments on the Analytic Hierarchy Process, Journal of the Operational Research Society, 41, 1073-1080. 
Johnson, C.R. (1980) Constructive critique of a hierarchical prioritization scheme employing paired comparisons, International Conference of Cybernetics and Society, IEEE, Cambridge, Mass.

Kotler, P. (1991) Marketing Management Analysis, Planning, Implementation, and Control, Prentice-Hall, New York.

Lootsma, F.A. (1989) Conflict Resolution via Pair-wise Comparison, European Journal of Operational Research, 40, 109-116.

Lund, J.R. and Palmer, R.N. (1986) Subjective Evaluation: Linguistic Scale in Pair wise Comparison Methods, Civil Engineering Systems, 3, 182-186.

McDonald, M.H.B. (1989) Marketing planning and expert systems: an epistemology of practice, Marketing Intelligence and planning, 7, 16-23.

McDonald, M.H.B. and Wilson, H.N. (1990) State-of-the-art development in expert systems and strategic marketing planning, British Journal of Management, 1, 159-170.

Mentzer, J.T. and Gandhi N. (1993) Expert systems in industrial marketing Industrial Marketing Management, 22, 109-116.

Mitchell, A.A., Russo, J.E. and Wittink, D.R. (1991) Issues in the development and use of expert systems for marketing decisions International Journal of Research in Marketing, 8, 41-50.

Rangaswamy, A. Eliashberg, J. Burke, R.R. and Wind J. (1989) Developing marketing expert systems: an application to international negotiations, Journal of Marketing, 53, 24-39.

Saaty, T.L. (1977) A Scaling Method for Priorities in Hierarchical Structures, Journal of Mathematical Psychology, 15, 234-281.

Saaty, T.L. (1978) Modeling Unstructured Decision Problems: The Theory of Analytical Hierarchies, Mathematics and Computers in Simulation, 20, 147157.

Saaty, T.L. (1980) The Analytic Hierarchy Process, McGraw-Hill, New York.

Saaty, T.L. (1990) How to Make a Decision: The Analytic Hierarchy Process, European Journal of Operational Research, 48, 9-26.

Salo, A.A, and Hamalainen, R.P. (1994) On the Meassurment of Preferences in the Analytic Hierarchy Process", Helsinki University of Technology Report, Finland. 
Wensley, A. (1989) Research directions in expert systems, in Knowledge-Based Management Support Systems, (ed. G.I. Doukidis, F. Land and G. Miller), Ellis Horwood, Chichester, 248-275.

\section{BIOGRAPHY}

Phillip Burrell is a Principal Lecturer and Director of the Knowledge Base Systems Centre at South Bank University, UK. He has researched in the area of Artificial Intelligence for many years and has been involved with a number of funded projects over this period. He studied at the University of Leeds, UK, Kings College, University of London, UK and undertook further research at Imperial College, University of London, UK.

Yanqing Duan is a Research Fellow in the faculty of Business at the University of Luton, UK and before that, a Research Fellow at the Knowledge Base Systems Centre at South Bank University, UK. She received her Ph.D. in Expert Systems from the Aston Business School, Aston University, UK. Her areas of research are with decision support systems and expert systems, especially for business applications .

Aleksandra Boskovic was a research student with LAFORIA-IBP, University Paris VI, France and participated in a European research student exchange program with the Knowledge Base Systems Centre at South Bank University, UK where she held a position as a research assistant. She is now pursuing a successful career in industry. 